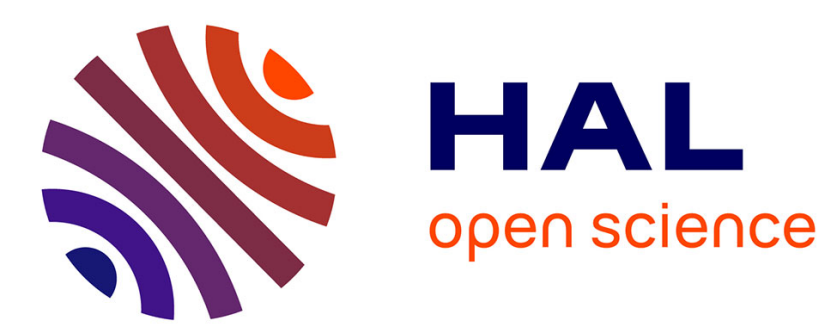

\title{
Représentation du territoire national et circulation des grains : le Système de distribution publique indien
}

\author{
Frédéric Landy
}

\section{To cite this version:}

Frédéric Landy. Représentation du territoire national et circulation des grains: le Système de distribution publique indien. Annales de géographie, 2011, 677, pp.26-49. halshs-01239990

\section{HAL Id: halshs-01239990 \\ https://shs.hal.science/halshs-01239990}

Submitted on 23 Nov 2021

HAL is a multi-disciplinary open access archive for the deposit and dissemination of scientific research documents, whether they are published or not. The documents may come from teaching and research institutions in France or abroad, or from public or private research centers.
L'archive ouverte pluridisciplinaire HAL, est destinée au dépôt et à la diffusion de documents scientifiques de niveau recherche, publiés ou non, émanant des établissements d'enseignement et de recherche français ou étrangers, des laboratoires publics ou privés. 


\title{
Représentation du territoire national et circulation des grains : le Système de distribution publique indien
}

\author{
Representation of national territory and grain \\ circulation: the Public Distribution System in India
}

Frédéric Landy

Professeur de géographie

Résumé En Inde, la pesante machine du Système de distribution publique (PDS) qui redistribue à prix subventionnés le grain ou le sucre achetés dans les zones de surproduction connaît de nombreux dysfonctionnements. Impératifs sociaux, soucis productivistes, intérêts illicites, nombreux sont les facteurs expliquant son maintien dans une Inde qui pourtant connaît de profondes réformes libérales. II en est un autre : dans son idéal, le PDS correspond en effet à deux représentations du territoire national qui se superposent en grande partie. La territorialité hindoue et son interprétation par la droite nationaliste, fondées sur les points cardinaux et sur leurs liaisons, tout comme la territorialité moderniste nehruvienne, fondée sur le souci d'intégration par des flux économiques, correspondent toutes à une conception de l'espace national basée sur la circulation, et qui concordent donc pour maintenir l'existence du PDS.

Abstract In India, the cumbersome Public Distribution System which sells at subsidized prices the grains and sugar procured in surplus regions is known for many flaws. Factors such as social needs, the productivist approach and vested interests help explain why the PDS persists in spite of the economic liberalisation and the partial withdrawal of the State. One missing factor is the fact that the ideal behind the PDS corresponds to two differing but superimposing conceptions of national territory. Both the (nationalist) Hindu territoriality, based on cardinal points and their linkages, and the modernist Nehruvian territoriality, based on a policy of integration created by economic flows, correspond to a circulationbased conception of the national space. Both are factors contributing to the maintenance of the PDS.

Mots clés Circulation, distribution alimentaire, flux, Inde, nation, territorialité.

Key words Circulation, flows, food distribution, India, nation, territoriality.

Plusieurs fois par an, aux moments des récoltes de blé, de riz, mais aussi de canne à sucre, les campagnes de l'Inde sont traversées d'un fourmillement de charrettes, de camions, de négociants en grains, de trains remplis de sacs de jute bourrés de céréales. Marchés « réglementés » au Punjab, huttes construites pour la pesée des grains au Tamil Nadu abritent portefaix, paysans, marchands, transactions multiples, commissions plus ou moins légales, couture à gros fil de sacs de $70 \mathrm{~kg}$. Rien de plus normal, pourrait-on dire, dans un pays où l'agriculture est encore pratiquée par la moitié de la population active. Pourtant, une bonne partie de cette agitation ne reflète nullement les activités du commerce privé 
et les logiques classiques de l'offre et de la demande : il s'agit en effet, pour un cinquième parfois de la production de blé et de riz, de flux institués à l'instigation de l'État. Une bonne partie des acteurs privés travaille de fait pour constituer des stocks publics de sécurité, qui puissent être redistribués à des prix subventionnés à la population indienne. Le système fonctionne parallèlement comme un soutien aux agriculteurs dans les zones de surproduction, qui ont grâce à l'État l'assurance de prix garantis. Certains trains circulent ainsi sur $3000 \mathrm{~km}$, du Punjab au Kerala, selon des itinéraires rendus complexes par la saturation saisonnière des infrastructures de transport (Landy, 2006).

Selon la National Sample Survey Organisation, en 1999-2000 12 \% des achats en blé et riz des ménages ruraux et $10 \%$ de ceux des urbains passaient par ce Système de Distribution Publique (PDS). Ces pourcentages ont pu frôler les $20 \%$ certaines années. Ils restent pourtant relativement faibles par rapport aux moyens mis en œuvre. De fait, le système est critiqué tant par la " gauche » (les ménages pauvres n’en bénéficient pas suffisamment) que par la «droite » (il est trop coûteux et entraîne des distorsions économiques). Dans un environnement mondial qui, OMC oblige, interdit de plus en plus une telle intervention de l'État, on peut donc se demander pourquoi ce système survit encore aujourd'hui . Les bonnes (et les mauvaises) raisons abondent, et sont souvent évoquées dans la littérature sur le sujet. Nous les rappellerons. Il en est une, cependant, qui n'est jamais évoquée : le rapport au territoire national indien. Sans jamais prétendre qu'il s'agisse d'un facteur majeur, ce texte montrera que la circulation des grains via le PDS correspond largement à une certaine représentation hindoue de l'espace, fondée sur la mise en relation des quatre points cardinaux. Un tel schème de pensée ne peut que faciliter la perpétuation d'un système pourtant attaqué de tout bord. C'est pourquoi, après avoir présenté en début de cet article le fonctionnement du PDS dans sa théorie comme dans sa pratique, il nous faudra évoquer la vision hindoue du territoire, dont les aboutissants ne s'opposent pas, loin s'en faut, à la vision nehruvienne et moderniste d'un espace à quadriller par des flux intégrateurs ${ }^{1}$.

\section{La machinerie publique alimentaire}

Le système est né, pour sa partie "distribution subventionnée », pendant la seconde guerre mondiale à Bombay et Calcutta. Il a progressivement été étendu à toutes les grandes villes de l'Inde, puis à toutes les petites villes et campagnes. Par les ventes des agriculteurs à l'État, le Punjab nourrit une bonne partie de l'Inde en blé, mais aussi en riz (fig. 1 et 2) malgré l'essor des achats publics pour cette

1 On parlera ici de " conception hindoue du territoire », en entendant par hindouisme une construction récente, et non une tradition remontant aux Vedas, renvoyant donc à la mobilisation d'un imaginaire religieux de la nation (N. Jaoul et K. Raj, communication personnelle). Par ailleurs, la place manque ici pour évoquer les représentations propres à chaque secte et chaque caste, sans parler de celles des Indiens non hindous. Merci à Kapil Raj, Marie Fourcade et Caterina Guenzi pour leurs remarques. 
dernière céréale en provenance de l'Andhra Pradesh. Cette extension spatiale du réseau traduit le souci de "chasser la pauvreté ", pour reprendre le slogan électoral d'Indira Gandhi, mais aussi un profond bouleversement de l'origine des grains. Jusqu'à la révolution verte, à la fin des années 1960, les stocks publics étaient alimentés en majorité par des importations. Il s'agissait avant tout de blé des États-Unis, en particulier à partir de la signature en 1956 d'un accord bilatéral sous l'égide de la Public Law 480. Pour le reste, une minorité de riz et de blé provenait des agriculteurs indiens, vendue le plus souvent sous la contrainte publique, ou des marchands, riziers et meuniers qui se trouvaient obligés de concéder à l'État une partie de leurs stocks à des prix peu élevés. Ces prélèvements (procurement) avaient une rigueur et une ampleur très variable selon les États et selon les années, mais d'une façon générale le système correspondait à une taxation des agriculteurs au profit de consommateurs avant tout citadins.

Tout change avec les succès de la révolution verte. L'Inde est à peu près autosuffisante en grains à partir de 1977, et, tout comme la Politique Agricole Commune européenne dont est contemporaine la révolution verte, elle se trouve en proie à un problème nouveau : maintenir des prix suffisamment attractifs pour le producteur afin de l'inciter à continuer à investir dans les intrants de la nouvelle technologie (semences améliorées, irrigation, engrais chimiques). Le PDS en sera le moyen : la Food Corporation of India (FCI), créée en 1965 lors du lancement de la révolution verte, achète désormais leurs grains aux agriculteurs, du moins pour ceux qui le souhaitent, à un prix relativement rémunérateur (même si le plus souvent inférieur au cours mondial). Le PDS intègre donc un volet productiviste qu'il a gardé jusqu'à aujourd'hui.

Le gouvernement fédéral s'adosse, comme pour toutes les questions relatives à l'agriculture, sur les États fédérés. Ceux-ci ont souvent leur propre politique d'achat, ajoutant parfois au prix fédéral un «bonus » afin de s'attirer la paix sociale dans les campagnes. Ils ont aussi parfois leurs propres stocks publics (Tamil Nadu), et se permettent parfois de «sur-subventionner » les prix dans les boutiques du PDS (ration shops). Certains États, dès les années 1980, établirent deux listes de prix pour le blé, le riz, le sucre ou l'huile subventionnés : une pour les ménages au-dessus du seuil de pauvreté, et une pour les ménages "pauvres » qui bénéficient tout à la fois de prix inférieurs et de quotas mensuels par personne supérieurs. Le gouvernement fédéral va imiter ce système en 1997, en espérant réduire la facture budgétaire du PDS : cette sélection permettrait, espérait-on, d'éloigner les ménages qui ne sont pas dans le besoin.

Ce fut l'inverse qui se produisit. Les ménages «non pauvres » n'achetèrent plus guère, certes, mais du coup les stocks de grain s'accumulèrent, et le prix de ce stockage fit plus que compenser les économies faites sur les subventions aux consommateurs. Le gouvernement fédéral se trouva même à la tête de 65 millions de tonnes de blé et de riz en juin 2002. Il dut exporter (jusqu'à 12,5 millions de tonnes de grains en 2002-2003), et revendre sur le marché intérieur. Mauvaise gestion ? En 2006 et 2007, ce fut le retour à des importations de blé quand, libéralisation du système aidant, les agriculteurs purent vendre au secteur privé 

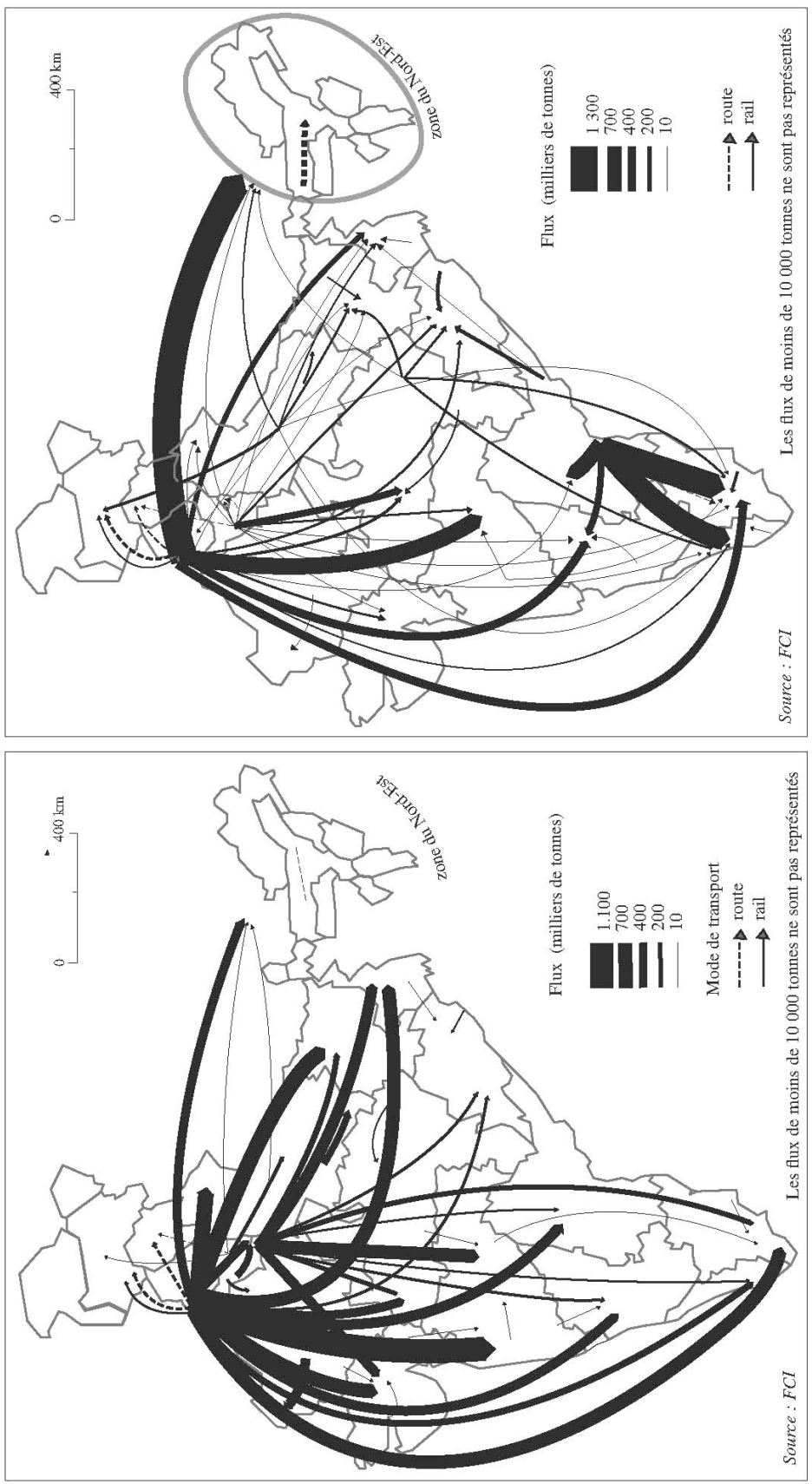

Source : Landy, 2006.

Fig. 1 Transport de blé et de riz par la Food Corporation of India en 1999-2000. Transportation of wheat and rice by the Food Corporation of India (1999-2000) 
(y compris aux multinationales, de plus en plus présentes), autrement attractif que l'État. Il existe donc des cycles composés de phases de surproduction, ou du moins de surstockage, alternant avec des phases de sous-disponibilité et d'importations. Dans le premier cas, la FCI est parfois obligée de vendre le grain aux négociants privés à des prix inférieurs à ceux concédés aux ménages pauvres, afin de favoriser les exportations et liquider les stocks (comme en 2002-2003). Dans le second cas, l'Inde doit importer à des prix supérieurs à ceux consentis aux agriculteurs par la FCI (comme en 2006-2007).

\section{Quelles raisons au maintien du PDS ?}

Les premières sont productivistes, on l'a vu. Il s'agit de maintenir l'intensification des systèmes de culture en prévenant tout engorgement des marchés dans les régions de surproduction. Mais certaines années les prix publics se trouvent bien plus bas que ceux du marché. Et les intermédiaires entre les agriculteurs et l'administration des achats publics (usuriers, marchands d'engrais, etc., qui vendent parfois avec des prête-noms la récolte à l'État) se partagent l'essentiel des soutiens.

D'autres raisons sont sociales. Aussi limitée qu'elle soit, la distribution subventionnée peut servir de bouée de sauvetage à bien des ménages miséreux. Cela dit, en 1999-2000, selon une enquête de la National Sample Survey Organisation, les ruraux pauvres économisaient ainsi $6 \%$ de leurs dépenses totales de consommation. Mais les citadins pauvres n'épargnaient que moins de $3 \%$, avec des achats de grain de $0,9 \mathrm{~kg}$ par personne et par mois... Quant à l'amont du système, qui vise à soutenir les prix agricoles, il est loin de favoriser tous les agriculteurs, puisqu'en Inde les quatre cinquièmes de ceux-ci achètent plutôt qu'ils ne vendent sur le marché étant donné la petitesse de leur exploitation.

Le seuil de pauvreté est par ailleurs défini fort bas, puisqu'il correspond en zone rurale à environ 11 roupies $(0,20 €)$ par jour de revenu pour un adulte, et à 19 Rs $(0,30 €)$ en zone urbaine ! Pourtant 60 millions de ménages indiens se situent sous ce chiffre qui fonctionne comme un seuil de misère plutôt que de pauvreté. Dans les mégapoles où les salaires sont relativement élevés, même les habitants de bidonvilles ne sont que rarement comptabilisés sous le seuil de pauvreté. Dès lors, à Mumbai seul le pétrole lampant est certaines années en vente dans les ration shops, étant donné que le rapport qualité-prix des céréales est trop faible pour intéresser des clients, désargentés mais officiellement «non pauvres ».

On devine donc que les raisons spatiales au maintien du système ne sont pas complètement convaincantes : il s'agit théoriquement d'approvisionner toutes les régions de l'Inde, y compris celles qui, trop éloignées des circuits commerciaux, ne pourraient bénéficier de distribution d'argent faute de grain physiquement disponible sur les marchés. Mais la réalité est autre. Les zones les plus déficitaires 
sont aussi celles où le PDS marche le moins, faute d'administration efficace et de développement suffisant.

Le Department of Food and Civil Supply du Bihar reçoit certes sa part de grains de l'État fédéral. Mais l'essentiel se perd au fil des transports et des ruptures de charge, sacs et même camions « disparaissant » vers le marché libre au profit d'une chaîne de corruption qui va du gérant de ration shop jusqu'aux hauts fonctionnaires et hommes politiques. Un rapport parlementaire annonçait en 1997 que $31 \%$ du riz, $36 \%$ du blé, $23 \%$ du sucre et $55 \%$ des huiles alimentaires n'atteignaient pas les bénéficiaires prévus ${ }^{2}$. Aux scandales médiatisés impliquant de grosses sociétés exportatrices répondent localement des myriades d'abus quotidiens, Intouchables ou veuves dont la ration est écoulée frauduleusement sur le marché libre, prélèvement d'un ou deux kilos de céréales sur les sacs acheminés aux boutiques par le gérant des entrepôts de canton, appels d'offres truqués pour le transport dans les camions privés, etc. Il faut bien l'admettre : corruption et prébendes sont des facteurs importants pour expliquer le maintien de la machinerie alimentaire de l'Inde.

Mais pourquoi, dans une ville comme Mumbai, des ménages sont-ils prêts à dépenser 5000 Rs en pots-de-vin pour obtenir la carte d'alimentation (théoriquement gratuite) qui ne leur servira qu'à acheter du pétrole (Kumar, Landy, 2009) ? Parce qu'existe un autre usage : celui de carte d'identité. Pour bien des démarches administratives, la carte d'alimentation est demandée. Elle fonctionne du même coup comme un certificat de résidence (en obtenir permet de limiter quelque peu les risques d'expulsion d'un bidonville), voire même comme une preuve de nationalité (en particulier pour les musulmans, souvent suspectés dans certaines régions d'être des immigrés clandestins du Bangladesh).

Aucune de ces raisons n'est, on le voit, satisfaisante à elle seule pour expliquer le maintien du système. Celui-ci résulte plutôt de leur accumulation. La littérature sur le sujet, fort abondante, en fournit de multiples illustrations et analyses (Mooij, 1999 ; Mahendra Dev et al., 2003). Pourtant, il faut ajouter un dernier facteur explicatif, qu'on qualifiera de territorial. Que la carte d'alimentation puisse fonctionner comme une carte d'identité atteste des liens entre PDS et nation. Mais ces liens valent également pour la «territorialité nationale », c'est-à-dire les rapports des citoyens indiens au territoire de leur pays - une territorialité qui traverse le PDS.

\section{Trois territorialités nationales}

Le $\mathrm{XX}^{\mathrm{e}}$ siècle aura vu se dégager deux territorialités à l'échelle de l'Inde, souvent complémentaires plutôt qu'incompatibles. Le premier de ces rapports à l'espace est religieux, et spécifiquement hindou. Le deuxième présida aux premières décennies de l'Inde indépendante, fondé sur la planification et la «modernité » :

2 Department of Food, Annual Report 2000-2001. 
on le qualifiera ici schématiquement de «nehruvien ». Il en est un troisième, « gandhien »- mais il n'a guère laissé sa marque dans l'aménagement du territoire ni a fortiori du PDS $^{3}$.

\subsection{La vision gandhienne}

La conception du territoire de Gandhi n'a jamais été présente au pouvoir à New Delhi (sinon occasionnellement, parmi certains membres du gouvernement Janata Party en 1977-1979) ni dans les politiques mises en œuvre (à part ces concessions que furent la politique de décentralisation de 1957 ou l'encadrement de la filière textile " traditionnelle» khadi). Ce point est essentiel car, la vision gandhienne se distinguant radicalement des deux autres, elle aurait pu considérablement changer la face du territoire indien et de son aménagement.

Or le Mahatma envisageait une nation fondée sur des cellules relativement autonomes, les villages. "Mon idée de l'indépendance des villages (village swaraj) est celle d'une complète république, indépendante de ses voisines en ce qui concerne ses besoins vitaux, et cependant interdépendante pour beaucoup d'autres quand cela est nécessaire. Aussi le souci de chaque village sera-t-il de faire pousser ses propres cultures vivrières et son coton pour ses vêtements » (Harijan, 26.7.1942, www.gandhiserve.org). Cette autonomie va de pair avec une décentralisation de la production et de la consommation peu compatible avec le grand dessein de la nation développée selon le schéma productiviste et moderne de Nehru. Le swaraj (souveraineté nationale) gandhien a pour aboutissement l'autonomie locale, au risque de l'émiettement, alors que la planification nehruvienne aura pour objectif l'intégration.

Pour Gandhi, le fagotage par des flux économiques du territoire national était un mal plutôt qu'un bien, source d'inégalités, de transactions corruptrices, d'oppressions sociales et de déclin moral. Gandhi refusait la modernisation de l'économie car elle allait de pair avec l'exploitation de la main-d'œuvre (Deshpande, 1993) : son idéal était l'absence d'échanges, du moins d'échanges faisant intervenir argent et salaires. Pour exister, la nation n'avait pas besoin d'un territoire irrigué par des flux de produits.

Il n'est donc pas très difficile d'imaginer ce que serait devenu le PDS si la conception gandhienne du territoire avait été au pouvoir. Sans doute l'alimentation des plus grandes villes serait-elle demeurée subventionnée quelque temps. Mais jamais l'extension du réseau ne se serait faite dans les campagnes. La bataille de l'autosuffisance aurait été menée à l'échelle régionale voire villageoise : aurait été refusée la logique propre à la révolution verte, de concentrer les investissements dans les régions les plus en avance car les plus susceptibles d'investir dans les nouvelles technologies agricoles. Il en serait allé de même

3 On schématise sous ces trois seules conceptions des courants de pensée peu homogènes. Plus de place serait nécessaire pour apporter nuances et complexifications, en particulier en ce qui concerne I'identification, sans nul doute schématique, entre territorialité hindoue et territorialité exprimée dans le discours de la droite hindoue. 
avec la politique de diffusion de la consommation de riz et surtout de blé dans toute l'Inde, qui se fit aux dépens des variétés et des espèces de céréales propres à chaque région (sorgho, éleusine, variétés locales de riz...). Gandhi postmoderne ? En tout cas, sa logique est en train d'être relancée actuellement par certaines ONG, telles que la Deccan Development Society ${ }^{4}$, qui tente en Andhra Pradesh de redynamiser les cultures de sorgho. On se trouve dans des logiques de développement local durable, qui doivent permettre aux défavorisés (femmes, zones sans irrigation, cultures oubliées par la recherche agronomique) d'acquérir une véritable self-reliance.

Gandhi était assurément hindou. Il ne nous semble pas toutefois que sa représentation du territoire national ait eu toutes les caractéristiques de ce qu'on qualifiera ici de "vision hindoue du territoire ", laquelle est communément considérée comme beaucoup plus globalisante et intégratrice que la représentation plus « éclatée » de Gandhi.

\subsection{La vision hindoue}

Analysons la représentation religieuse du territoire indien en la décomposant en deux sous-types de territorialité : statique avec les quatre points cardinaux, circulatoire pour relier ces points.

\subsubsection{Les points cardinaux}

Ils sont fondamentaux pour la représentation du territoire hindou national. Leur signification géopolitique était présente dès l'époque antique. Ainsi, la "conquête des quatre orients»(dig-vijaya) est une thématique récurrente dans les hagiographies de saints comme dans les épopées guerrières et royales. Le roi comme le saint doit dominer les quatre directions pour asseoir sa domination. (Aujourd'hui encore, Digvijay est un prénom usuel.) Au VIII ${ }^{\mathrm{e}}$ siècle, le théologien Sankara, "conquérant des quatre orients », installe ses centres aux quatre directions de l'Inde: Dwarka, Badrinath, Puri et Sringeri. À la fin du $\mathrm{XIX}^{\mathrm{e}}$ siècle encore, Vivekananda implantait ses quatre principales missions aux quatre points cardinaux - Madras, Calcutta, Allahabad et Bombay.

Il importe certes de ne pas exagérer l'importance de ces directions pour le bornage de l'Inde. (Il s'agit d'une façon générale de ne pas essentialiser ce qui est parfois douteux historiquement - mais qui demeure extrêmement prégnant dans le domaine des représentations et des discours contemporains). Dans l'espace hindou reconnu par la plupart des croyants, seules les « demeures » divines (dham) - les quatre villes de Dwarka à l'ouest, Puri à l'est, Badrinath ou Kedarnath au nord, et Rameshwaram au sud - correspondent aux quatre points cardinaux. Pour le reste, le territoire est plutôt quadrillé d'une multitude de lieux sacrés, dispersés à travers le territoire sans ordre spatial véritable. Ce sont cependant souvent des ensembles unifiés par un mythe unique (Feldhaus, 2003) : ainsi des quatre sites de Kumbha Mela dont le pèlerinage, tous les douze ans, attire des millions de 
pèlerins sur quelques jours; ou des douze linga de Shiva nés du sperme divin (jyotirlinga). Autant de lieux qu'on parcourt comme pèlerin, ou du moins qu'on nomme couramment dans le discours populaire, et qui très tôt, au temps où l'Inde n'était que mosaïque d'États princiers, de nababs et de rajas, servirent à unifier le pays sous une foi spatialement définie.

Le discours commun intègre couramment les points cardinaux pour évoquer le territoire hindou - et même, nous le verrons, le territoire indien. Les quatre directions fonctionnent ainsi comme une véritable métaphore de l'Inde. Et l'on glisse alors facilement d'une «territorialité hindoue » à une territorialité "nationaliste hindoue ». Ainsi, V. D. Savarkar, l'auteur du fameux Hindutva (Hindonité, publié en 1923) et un des fondateurs du mouvement nationaliste hindou, n'est pas le premier à cartographier dans son livre le territoire hindou "from Kashmir to the Cape [Comorin] and from Attock [Punjab pakistanais] to Cuttack [Orissa] »(Savarkar, 1969, p. 79 et passim). Il ne fait que reprendre des expressions toutes faites plus anciennes, qu'on trouve dès les années 1870 dans les livres scolaires ou la presse (Goswami, 2004). Une espèce de signe de croix hindou... cité cependant chez Savarkar dans un sens inhabituel, puisque dans les textes védiques l'ordre est plutôt: est, ouest, nord, sud. C'est une énumération utilisée alors aussi bien juridiquement, pour délimiter les propriétés foncières, que, dans les épopées, pour indiquer que le héros contrôle l'ensemble de son territoire (Gaborieau, 1992). Ce qu'ajoute Savarkar à cette manière de cartographier le territoire, ce sont les allitérations ( from Attock to Cuttack»). Les échos des consonnes, les résonances des syllabes que se renvoient les mots sont comme la preuve de l'unité de l'Inde, dont les quatre coins réfléchissent l'identité multiforme.

Ne nous étonnons pas que ceux-ci soient si visibles dans la toponymie indienne. La figure 2 montre que quatre États de l'Union portent dans leurs noms une référence aux directions. Seul l'ouest n'est pas représenté. Est-ce un hasard ? Borner aussi cette direction reviendrait à entériner la perte du Pakistan et à accepter définitivement la Partition ; pour beaucoup d'Indiens dans les années 1950, la plaie n'était pas encore assez cicatrisée pour accepter par la toponymie que le Gujarat ou le Jammu \& Kashmir deviennent les limites occidentales de l'Inde indépendante.

\subsubsection{Le parcours : croix et chakra}

Le second type de territorialité hindoue est circulatoire. Il s'agit cette fois de parcourir le territoire, physiquement ou par la pensée. Deux types d'itinéraire alors : soit relier les quatre points cardinaux deux à deux, à la façon d'une croix ; ou bien les relier "par la bande ", de façon circulaire.

Le premier type est particulièrement récurrent à travers le cas des processions. On trouve trace dès 1911 de processions nationalisto-religieuses où l'on promène des images de Mère Inde (Bharat Mata) devant la carte du pays (Goswami, 2004). Cette déesse, popularisée à la fin du XIX ${ }^{\mathrm{e}}$ siècle par l'écrivain nationaliste et hindou Bankim Chandra Chatterji, correspond à une figure féminine et 


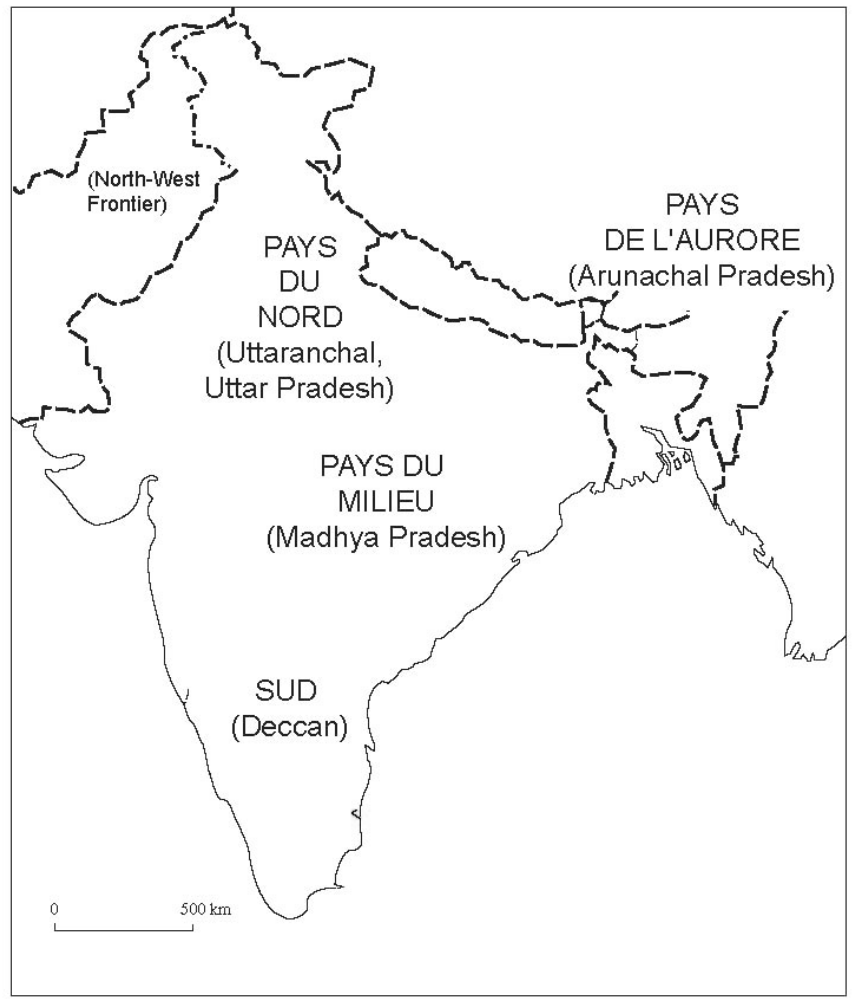

Fig. 2 La toponymie officielle marquée par les quatre directions The official toponymy includes many cardinal points.

maternelle très présente dans le discours contemporain de la droite hindoue (Assayag, 2001 $)^{5}$. Elle n'a aujourd'hui que peu de temples consacrés dans toute l'Inde, mais elle demeure très populaire et représentée sous forme de vignettes, autocollants, calendriers: femme guerrière accompagnée d'un tigre, elle est toujours montrée debout, depuis sans doute 1909, devant une carte de l'Inde - une carte sans frontières bien définies, ce qui permet d'intégrer la Grande Inde dont rêvent certains nationalistes hindous (Ramaswamy, 2006 ; Claveyrolas, 2010). La popularisation de ce "nativisme territorial » va grandir au cours du $\mathrm{XX}^{\mathrm{e}}$ siècle à travers des processions, tournée de plus en plus à l'encontre des nonhindous plutôt qu'à l'encontre des non-Indiens : c'est ce que la droite hindoue va appeler des "pèlerinages patriotiques ». Mère Inde - ou bien le dieu Rama - sont en effet affichés comme "sans caste » et vénérés par tous les hindous. Il

5 Chez les nationalistes hindous, le territoire de la nation est donc plus souvent représenté en termes anthropomorphes (Brosius 2009, Ramaswami 2006) qu'avec les points cardinaux. Ceux-ci sont cependant présents dans le discours. 
convient de protéger ce territoire menacé par les ennemis de l'intérieur comme de l'extérieur. Dans les vidéos de propagande qui circulent à cette occasion (Brosius, 2009), on peut voir ainsi des animations montrant une carte de l'Inde en cours de désintégration, perdant ses extrémités géopolitiquement les plus fragiles : Punjab, Assam, Kashmir - l'ouest, l'est, le nord ${ }^{6}$.

Les processions ont culminé à partir des années 1980. Leur parcours reprend souvent un itinéraire à forme de croix reliant les points cardinaux (Assayag, 2001), et centré sur Nagpur-Ramtek, à la fois centre géographique de l'Inde et siège du mouvement extrémiste RSS (Landy, 2006). Ainsi, la procession commencée par le ministre de l'Intérieur L.K. Advani en mars 2004 (fig. 3), à la veille d'élections générales qu'allait perdre son parti BJP, a démarré en autocar de Kanya Kumari pour rejoindre Amritsar (le Kashmir était sans doute jugé comme trop dangereux ou provocant), puis dessiner la seconde branche ouest-est de la croix, « de Porbander à Puri ${ }^{7}$ »- encore une allitération.

C'est à l'échelle d'une agglomération comme du pays tout entier que se forment alors deux cortèges ou deux itinéraires qui se coupent grossièrement en leur moitié. La croix peut même parfois avoir la forme de svastika ${ }^{8}$. En témoigne la figure 4 de la procession en l'honneur de Ganesh qui eut lieu à Hyderabad en 1980 (Naidu, 1990). Quatre branches convergèrent d'abord vers le Char Minar ( «les Quatre Minarets»), au centre de la vieille ville habitée en majorité par une population islamique. Il s'agissait de provoquer les riverains (la procession était organisée par les mouvements nationalistes hindous du VHP et du RSS ${ }^{9}$ ), mais aussi de dessiner dans l'espace la convergence vers un centre en provenance des quatre points cardinaux : les cortèges traversèrent des quartiers musulmans comme des quartiers hindous, par souci géométrique comme par provocation. (Au contraire, la procession de la fête de Muharram, représentée elle aussi, a un tracé qui ne relève pas de la croix, et un itinéraire limité à certains quartiers chiites de la vieille ville). Le cortège ensuite a traversé la rivière Musi pour aller, au nord (une direction favorable), immerger des statues du dieu dans le grand lac intra-urbain Hussain Sagar - rebaptisé en l'occurrence «Vinayak Sagar » par les nationalistes hindous pour effacer un nom trop teinté d'islam ${ }^{10}$.

6 II manque le sud. Assez curieusement, la question tamoule au Sri Lanka n'est à ma connaissance jamais mentionnée par les nationalistes hindous, alors qu'elle pourrait représenter le « front sud » de la lutte.

7 Frontline, 9 avril 2004, p. 123.

8 Symbole hindou de chance, repris par les nazis pour en faire en l'inversant la croix gammée.

9 VSP : Vishva Hindu Parishad, Association Hindoue Universelle. RSS : Rashtriya Swayamsevak Sangh, Association Nationale des Volontaires.

10 La fête de Ganesha célébrée à Hyderabad le 17 septembre 2005 donna lieu un autre circuit sous forme de croix, dont les branches convergeaient cette fois directement vers le lac de Hussain Sagar pour y immerger 15000 statues du dieu à tête d'éléphant. La branche venue du sud fut la première à démarrer, peut-être parce qu'il s'agit d'un point cardinal néfaste, sûrement aussi parce qu'elle devait traverser la vieille ville musulmane et qu'il s'agissait que le parcours soit achevé avant que la nuit tombe. Les branches ouest et est suivirent, tandis que la branche nord fut la dernière à rejoindre le lac (Deccan Chronicle, 17.9.2005). 


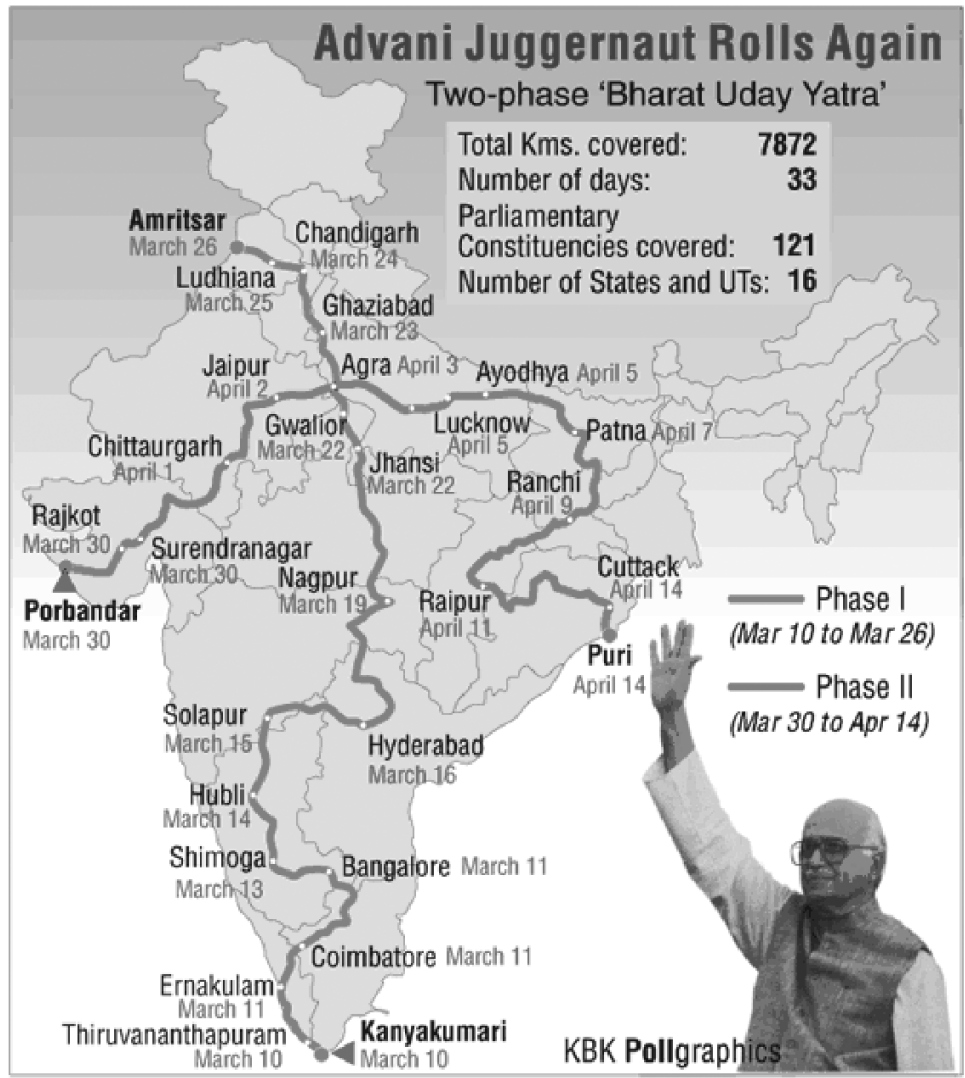

Source : http://in.rediff.com/news/yatra04.htm

Fig. 3 La «procession de l'Inde qui brille» (Bharat Uday Yatra) de L.K. Advani en 2004. The « Shining India procession » of L.K. Advani in 2004.

Une autre forme de territorialité hindoue consiste à relier les points cardinaux par un cercle - parfois un quadrilatère. Cette circumambulation, très courante à l'échelle locale (dans les temples, pour célébrer les mariages, etc.), l'est aussi aux échelles supérieures : elle est utilisée pour relier entre eux les temples visités lors des processions, dans le sens des aiguilles d'une montre, à l'intérieur ou à la périphérie de Bénarès (Gaenszle, Gengnagel, 2006 ; Claveyrolas, 2010). Mais il reste à prouver que les circuits organisés de visite des quatre villes sacrées (char dham) se font toujours dans ce sens favorable.

Si le cercle de la circumambulation est combiné avec la forme précédente - la croix -, on obtient une forme de roue, dont les rayons forment croix (ou étoile 


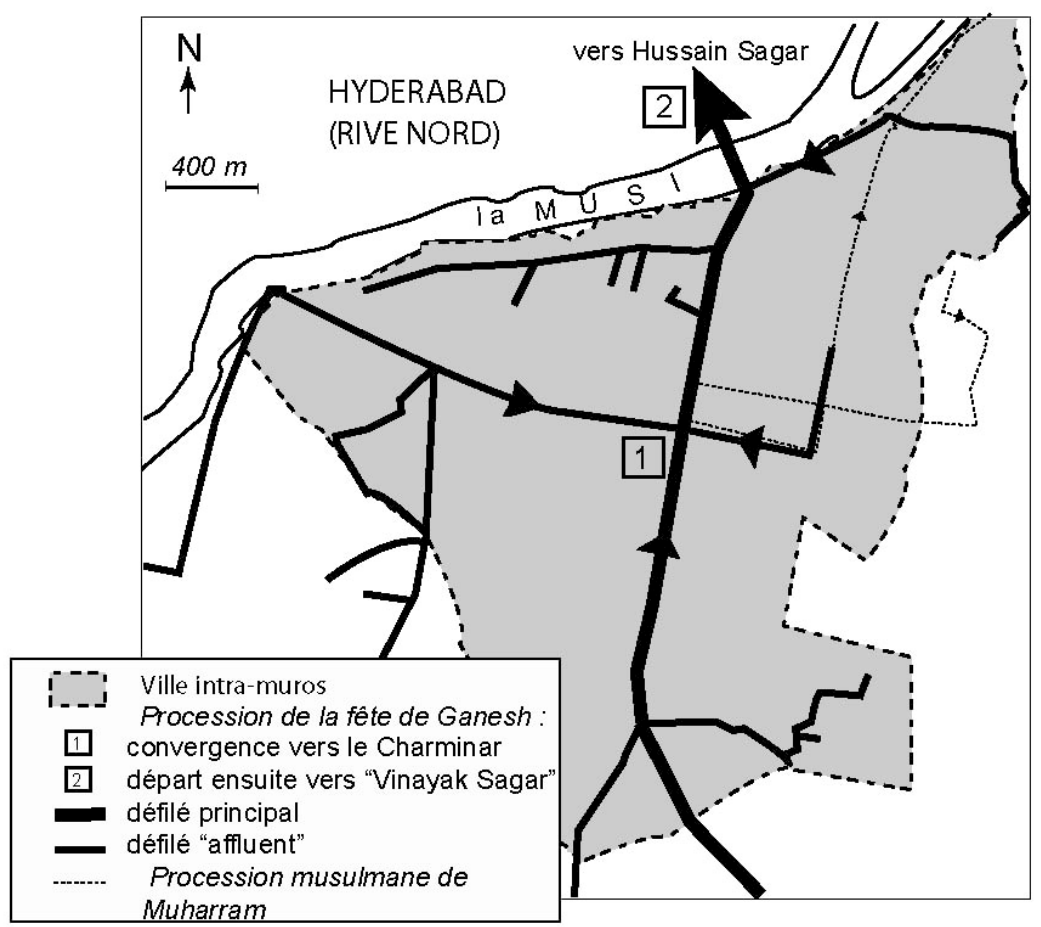

Source : d'après Naidu, 1990.

Fig. 4 Processions religieuses dans la vieille ville de Hyderabad (1980).

Religious processions in the Old City, Hyderabad (1980)

s'ils sont plus nombreux que quatre). On reconnaît alors un éminent symbole hindou, le chakra.

Le chakra est l'arme redoutable que lance le dieu Vishnu. Il est un symbole de l'empereur Ashoka. La roue est symbole de pouvoir. Chakravarthy, un nom propre très répandu en Inde, est à l'origine un terme désignant le monarque : "celui qui fait tourner le chakra», la roue du dharma ${ }^{11}$, autrement dit qui gouverne selon la loi ; mais aussi dans un sens plus matériel «celui qui a fait circuler son char (de guerre) sur tout son royaume », le souverain qui parcourt les limites de son territoire pour mieux le contrôler (Fussman, 1980 ; Bruneau, 2006). Le contrôle du territoire se fait en parcourant sa superficie, en reliant les quatre directions, mais à partir d'un centre qui se trouve être comme le moyeu de la roue, d'où partent les rayons, et qui relie à un axe vertical - vers le ciel et l'enfer. 
La roue est aussi un symbole économique : elle est le rouet (charka) cher à Gandhi. Elle est l'engrenage, symbole de Vishvakarma le dieu des artisans ; le moyeu de bien des volants et machines d'usine est marqué de vermillon par les ouvriers pieux (Lachaier, 1999). La roue dharmique pouvait même être vue au plafond de la salle de marché de la Bourse de Bombay, où elle était décrite par les Indiens comme un bub - terme éminemment polysémique (Lachaier, à paraître). Industrie, politique et religion se mêlent donc dans la roue dessinée sur le drapeau national indien, qui est tout à la fois chakra et charka ${ }^{12}$.

Si l'on se livre à nouveau au petit jeu de l'uchronie, et qu'on imagine un moment Savarkar et la droite hindoue au pouvoir dès les années 1950, quelle machinerie alimentaire publique aurait pu être créée ? Répondre à cette question est plus difficile que celle portant sur la territorialité gandhienne. Tout ce qui précède peut donner l'impression que l'extension progressive du PDS correspondait parfaitement aux schèmes de pensée des hindous en général, et des nationalistes en particulier : le souci de faire circuler des flux de pèlerins, ou de militants, aux quatre coins du territoire pour mieux se l'approprier répond à un modèle de réseau très proche de celui du PDS. On comprend alors que le BJP, au pouvoir fédéral de 1998 à 2004, n'ait pas contre toute attente démantelé le PDS alors qu'il était pourtant tenant d'une politique globalement libérale. Intérêt électoral de ne pas se couper des populations pauvres, souci d'une politique agricole et sociale furent des facteurs sans doute essentiels au maintien du PDS. Mais l'on peut penser que la représentation du territoire national en fut un autre.

Cependant, il est vraisemblable que, la droite hindoue fût-elle au pouvoir dans les années 1950-1970, le système alimentaire public n’aurait pas été développé de la façon dont il le fut. Certaines des conceptions des nationalistes hindous ne sont en effet pas si éloignées de celles de Gandhi. Eux aussi tendent à se défier de l'État, prolongeant une vieille tradition brahmanique de méfiance envers le pouvoir temporel. Comme voulait le croire en 1965 un idéologue du RSS, D. Upadhyaya, « dans notre contexte socio-politique, le roi et l'État n'ont jamais été considérés comme suprêmes. [...] Le plus puissant des rois n'interférait jamais avec les panchayats [...]. L'État ne se préoccupait donc que de certains aspects de la vie de la société13 ${ }^{13}$. Reprenant l'utopie des villages unis des anciens temps, les nationalistes hindous rejoignent du même coup les gandhiens dans un discours prônant la décentralisation. Sans jamais penser à une éventuelle contradiction, le discours d'Upadhyaya tonne contre une Constitution trop fédérale qui ne rend pas compte de l'indivisibilité de la patrie, mais propose par ailleurs que les

12 Gandhi voulait que le rouet (charka) soit dessiné sur le drapeau national, et non le chakra, disque denté pour lui symbole de violence. Nehru réussit à le faire céder, en précisant devant la Constituante que sur le drapeau, " la roue bleu marine représente le charka, mais le dessin de la roue sera celui du chakra d'Asoka » (22 juillet 1947, cité par L.S. Rengarajan, "Fracas over the Flag », The Hindu, 15 août 2004).

13 www.bjp.org/history. 
provinces, les petites régions historiques (janapada) et les panchayats bénéficient d'une forte décentralisation de l'autorité.

Parions donc que la droite hindoue aurait été réticente (mais non fondamentalement hostile) à l'extension nationale du PDS. Sans doute revenait-il à la représentation nehruvienne du territoire de présider au développement de la machine alimentaire publique.

\subsection{La vision nehruvienne}

Le nehruvisme - appelons ainsi la vision dominante de l'Inde indépendante, secular (laïque) et progressiste - porte avec lui son paradigme territorial.

À l'Indépendance de 1947, faire survivre et prospérer la jeune Inde demeurait un défi. Il fallait perpétuer «le passage d'une société où l'autorité provenait de diverses manières locales à une société où elle se situait dans un pouvoir souverain unique », avec « la revendication de gouverner sur un territoire défini » (Khilnani, 1999 , p. 3 et 21). L'ampleur de la tâche s'évalue en revenant quelques années en arrière.

La Découverte de l'Inde : cet ouvrage à titre de guide de voyage fut écrit par Jawaharlal Nehru pendant les trois années qu'il passa en prison, de 1942 à 1945. On pourrait attendre de cet avocat anglophone, bourgeois et brahmane, ancien étudiant de Cambridge, une sorte de journal de voyage où l'auteur conterait les progrès de sa tardive connaissance de l'Inde au hasard des routes. Il ne s'agit pourtant point de cela. Il n'est pas question de géographie, mais d'histoire. Il s'agit de forger le nationalisme en exhumant les fondements de la nation. Nehru croit aux leçons de l'histoire et a pour mission de les livrer.

Le livre, à son début, est cependant traversé par de brèves mentions de périples à travers l'Inde. Ce sont ces voyages qui font germer les interrogations de Nehru à propos du passé et du devenir de son pays. Ce sont les lieux qui permettent d'écrire l'histoire, en posant des questions au voyageur. En cela, Nehru suit d'une certaine façon la démarche de la géographie classique, mais en malmenant le diptyque «description - puis explication » par une description trop brève, et par une explication trop exclusivement historique. La campagne électorale de 1936-1937 qu'il décrit rapidement, ses voyages " avant tout en automobile, partiellement en aéroplane et en train ", mais aussi en éléphant, en chameau, à cheval, en canoë ou à vélo, cette découverte de l'Inde par les yeux et les jambes peuvent être considérés comme formateurs du nationalisme nehruvien ${ }^{14}$. Peut-être même est-ce parce que l'espace est premier - donc évident - qu'il occupe si peu de place dans l'œuvre. Toujours est-il que Nehru affirme faire référence à ce territoire national dans ses discours politiques auprès des masses rurales, quand, pour évoquer « les problèmes communs aux paysans du nord au

14 Premier ministre, Nehru "pratiquait » à tel point son territoire que pour les élections de 1952, il parcourut 18000 miles en avion, 5200 en voiture, 1600 en train et 90 en bateau. " Un fonctionnaire du parti épuisé compara ensuite ceci "aux campagnes impériales de Samadragupta, d'Ashoka et d'Akbar" »(Guha, 2005, p. 1958). 
sud et d'est en ouest", il leur " raconte ses voyages du col de Khyber dans le lointain nord-ouest jusqu'à Kanya Kumari ou Cap Comorin dans le sud distant» (p. 60).

Ce repérage dans l'espace à partir des quatre points cardinaux est le même que chez Savarkar et les nationalistes hindous. On le retrouve aujourd'hui, traduit matériellement, dans ces grandes cartes murales qui ornent l'arrière des bureaux dans l'administration, symbolisant le pouvoir de ces hommes tout comme l'étendue de leur tâche. Aujourd'hui encore, les hauts fonctionnaires de New Delhi ont une expression toute faite à la bouche pour évoquer l'Inde entière : « de Srinagar à Kaniya Kumari ».

"La tâche n'était pas aisée », continue Nehru pour évoquer ses contacts avec les paysans ; «ce n'était pourtant pas si difficile que je l'imaginais, étant donné que nos épopées, nos mythes et nos légendes antiques, qu'ils connaissaient si bien, leur avaient rendu familière cette conception de leur pays, et il y en avait toujours quelques-uns qui avaient voyagé aux quatre coins de l'Inde à destination des grands lieux de pèlerinage »(p. 60). Ces pèlerinages représentent à l'époque, encore davantage qu'aujourd'hui où il existe d'autres modes d'identification, une circulation soudant le pays "dans un rituel d'intégration nationale » (van der Veer, 1994, p. 124). Les musulmans font de même, pourrait-on ajouter, puisqu'à La Mecque s'ajoutent pour eux bien des pèlerinages spécifiquement indiens, en particulier Ajmer et, à Delhi, Nizamuddin. Nehru le redit plus loin dans son livre : «On fit une tentative marquée et assez réussie pour créer un lien national commun qui puisse maintenir ensemble tous ces groupes - le sens d'une culture commune, de traditions communes, de héros et de saints communs, et d'une terre commune aux quatre coins de laquelle les gens partaient en pèlerinage. Ce lien (bond) national était bien sûr fort différent du nationalisme d'aujourd'hui ; il était faible politiquement, mais, socialement et culturellement, il était fort » (Nehru, 1985, p. 251).

On l'a vu : le drapeau de l'Inde laïque (secular) affiche un symbole, charka ou chakra, qui peut être lu religieusement. Et le nom officiel de l'Inde, Bharat, est un nom provenant d'une épopée hindoue, le Mababharata. On est passé insensiblement du domaine religieux à celui du politique. Autant d'ambiguïtés dont avaient plus ou moins conscience les fondateurs de l'Union indienne. Pour eux, l'unité de la nation indienne était trop récente et trop fragile pour qu'on ne s'appuie pas sur l'unité, bien plus consolidée, de la nation hindoue. Quitte à bien sûr la sculpter, la modifier, afin qu'elle soit véritablement intégratrice et accueille en son sein toutes les cultures, et toutes les religions. Aujourd'hui, on peut penser que c'était «jouer avec le feu».

Ramaswamy (2006) a bien montré que si le temple de Bharat Mata de Bénarès, inauguré en 1936 par Gandhi, contient pour toute statue à vénérer une carte en relief de l'Inde, en marbre, c'est pour pouvoir réunir dans une même foi nationaliste toutes les castes et toutes les religions de l'Inde : l'apport d'une cartographie scientifique et coloniale à la construction de ce qui reste un temple apparaît comme emblématique de cette combinaison de deux cultures qu'on 
retrouve dans La découverte de l'Inde. Nehru parle à ces paysans en des termes quasi religieux. Il évoque avec eux Bharat Mata, personnalisation de l'Inde sous forme d'une déesse - à peine moins laïque que la Marianne française dont elle est à peu près contemporaine ${ }^{15}$. L'objectif est de renforcer ce que Tuan (1976) appelle la "géo-piété », l'amour du territoire.

C'est à une conception hindoue du territoire que le laïc Nehru se réfère, non seulement à des fins très utilitaristes (convaincre un auditoire), mais aussi parce que celle-ci correspond presque en fin de compte à sa propre territorialité, moderniste et marxisante. L'Inde indépendante ne peut connaître " l'unité dans la diversité ", pour reprendre un slogan couramment utilisé, que si cette diversité est maintenue fagotée, attachée par les liens du développement et du progrès. Ce territoire national, il faut le doter de canaux et d'usines sources d'unité, non de temples et de mosquées sources de division. Il faut rattacher au mainstream, à l'Inde qui progresse, les espaces et les groupes sociaux à l'écart, tout en favorisant la croissance nationale : le premier point servira au second - et non l'inverse -, c'est-à-dire que priorité est donnée à l'échelle de la nation, aux dépens éventuels des intérêts des populations locales. L'aménagement du territoire (regional planning) se fera au bénéfice de l'Union : c'est pour l'autosuffisance alimentaire ou sidérurgique de la nation qu'on bâtira dans une zone tribale un nouveau barrage " temple de l'Inde moderne » ou une aciérie. Dès lors, il importe de strier le territoire national de flux économiques de toutes sortes pour mieux souder la fontanelle du jeune pays, y compris jusqu'à ses extrémités les plus lointaines : c'est là que la conception nehruvienne rejoint la conception hindoue du parcours du territoire.

La vision politique de l' «Union » indienne est très tôt une vision économique, qui se trouve en partie fondée sur la circulation. Comme l'a souligné Deshpande (1993, p. 16), « une des façons concrètes dont l'économie a pénétré les représentations nationalistes, et a en fait contribué à les façonner, se fit par l'intermédiaire des marchandises ". C'est à cette époque qu'on prend tout à fait conscience que tel tissu vient d'Angleterre ou est au contraire swadeshi, et que les sacs de grain vendus sur un marché du Punjab peuvent se trouver écoulés dans toute l'Inde voire au-delà. La circulation des produits commercialisés tend à définir des territoires qui vont de plus en plus être qualifiés de « national » ou d'étranger.

Que la planification aille dans le même sens qu'une conception religieuse convenait fort bien à la vision nehruvienne du territoire, à laquelle correspondait une politique d'aménagement menée par un personnel fondamentalement laïc mais en majorité hindou. Les deux conceptions pouvaient ainsi se superposer dans le même souci d'intégration spatiale de la nation, et permettre ipso facto l'extension progressive du PDS sur plus de quatre décennies (Landy, 2006).

15 «Marianne » est née sous la Révolution, mais n'a été véritablement consacrée comme symbole officiel de la République que sous la $3^{\mathrm{e}}$, en pleine période de culte marial. Son nom est composé de ceux de la mère et de la tante du Christ. Sa laïcité est donc pour le moins teintée d'ambiguïté. 
Des trois conceptions du territoire que l'on a analysées, celle de Gandhi est la plus statique, indifférente voire même hostile à la circulation. Celle de Nehru est au contraire comme fondée sur la circulation, car l'Inde y est un système dynamique donc instable, en permanence à réinvestir et à intégrer par des flux de toutes sortes. Celle de la droite hindoue représente un cas intermédiaire, puisqu'il s'agit d'un modèle circulatoire, mais non établi sur des bases d'économie politique comme celui de Nehru. Ces deux dernières vont cependant toutes deux dans le sens d'une forte intégration par la circulation du territoire national. Le PDS en a tiré profit.

\section{$4 \quad$ L'étoile et le PDS}

Au final, il n'est pas toujours aisé de savoir ce qui, de la conception hindoue ou de la conception (post)nehruvienne, est responsable de certaines politiques territoriales. L'Inde contemporaine donne de nombreux exemples de formes spatiales « hindoues » correspondant à des politiques « laïques".

Quand ce ne sont pas les quatre points cardinaux qui sont pris en compte, mais les multiples directions intermédiaires, la croix se transforme en une étoile à nombreuses branches. Elle peut alors correspondre à des flux centrifuges ou centripètes. Pour les flux centrifuges, citons les morts de la guerre de Kargil contre le Pakistan, en 1999, dont les dépouilles seront transportées à Delhi, puis dans les capitales provinciales, avant d'être rendues aux familles dans le cadre de funérailles organisées par l'État (Zins, 2005). Citons aussi un projet spécifiquement nationaliste hindou, du mouvement VHP : bâtir un temple à Pokhran, sur le site des essais de la bombe atomique indienne en 1998, donc lieu de fierté nationale à quelques kilomètres de l'ennemi musulman pakistanais, puis prélever du sable radioactif pour le promener en processions dans tout le pays... En ce qui concerne les mouvements centripètes, on a évoqué les pèlerinages majeurs qui font converger de l'Inde entière des centaines de milliers de personnes vers les sources du Gange ou les grands confluents ( 70 millions » pour le Kumbh Mela de Nashik en 2003, selon Wikipedia...). Ceux qui ont pour destinations des marges géopolitiquement fragiles mêlent les deux conceptions du territoire, hindoue et nehruvienne. Ainsi, le pèlerinage au grand linga de glace de la grotte d'Amarnath concerne une zone du Ladakh accessible par le Kashmir: l'État indien n'est pas le dernier à affirmer que "yearning for moksha (salvation) can move the devotees to the challenging heights of Kashmir and will be a fitting gesture of solidarity with our valiant soldiers who have been fighting the enemy to defend our borders ${ }^{16}{ }^{\prime}$. Le pèlerinage permet de contrôler un territoire, religieusement comme (géo)politiquement. Étant donné qu'il existe des partis politiques à base religieuse, la confusion est de toute façon inévitable. En plus des grandes

16 Communiqué du très officiel Press Information Bureau cité par Navlakha (2006) (http://pib.nic.in/feature/fe0799/f1507992.html). 
processions nationalistes hindoues qui convergent vers le centre géographique de l'Inde, il faut évoquer ces briques (flux de produits, et non plus d'hommes) expédiées de tout le monde hindou, y compris hors d'Inde, pour contribuer à la construction d'un temple de Rama à Ayodhya, sur le site de l'ancienne mosquée détruite en 1992 par des militants hindous.

Quand cette étoile est combinée à un cercle, elle forme une roue. Cette forme idéale de chakra est évidemment rarement accessible dans la réalité. La planification indienne y est cependant parvenue dans au moins un cas : le projet de construction d'autoroutes lancé en 1999 (fig. 5). Celui-ci, extrêmement volontariste, relie sans surprise les quatre plus grandes villes de l'Inde - c'est le cercle de la roue. Mais plus audacieusement, car sans visées économiques directes, il relie aussi les quatre points cardinaux deux à deux, formant ainsi les rayons de la roue, pour joindre des régions peu dynamiques. Un peu comme si, en France, on avait commencé le réseau autoroutier par une liaison de Brest à Strasbourg, et de Lille à Perpignan. Il est paradoxal que l'Inde, au moment où décline la planification, ait mis en route un tel programme d'aménagement du territoire. Visiblement, les nécessités économiques n'imposaient pas cette croix autoroutière ; les nécessités géopolitiques ne la suggéraient guère ; les nécessités de rééquilibrage régional un peu plus; mais il faut sans doute faire intervenir en fin de compte la représentation hindoue du territoire, combinée à la conception nehruvienne, pour avoir tous les facteurs en main expliquant " one of the most ambitious projects launched in independent India ${ }^{17}$ ».

Quant au projet d'interconnexion des fleuves de l'Inde, relancé à la même époque, qui pourrait permettre d'accroître la disponibilité en eau (ce que certains contestent) au prix d'énormes conséquences écologiques, sociales et financières (ce que beaucoup affirment), il ne se traduit certes pas par une carte aussi spectaculaire que celle des autoroutes ${ }^{18}$. C'est que la gravitation universelle ne permet pas à l'eau de remonter les pentes, et qu'un déterminisme physique empêche ici de laisser libre cours au même volontarisme en matière d'aménagement du territoire que pour la construction d'autoroutes.

Tous ces projets ont été lancés pendant la période où les nationalistes hindous étaient au pouvoir. Ils « sonnent » pourtant comme des projets nehruviens : même État démiurge, même souci de l'échelle nationale, fût-ce aux dépens des intérêts locaux, même culte de la croissance économique. L'architecte Jeet Malhotra, appartenant à la mouvance nationaliste hindoue tout comme à l'héritage de Le Corbusier, pouvait encore publier en 2000 dans la revue indienne Architecture + Design (vol. 17, 1) un projet pharaonique quadrillant géométriquement le territoire indien par des axes industriels et urbains rigoureusement orthogonaux. Tout cela alors que le gouvernement n'avait de cesse d'afficher des mesures allant dans le sens du libéralisme.

17 10th Plan, box 8.3.4.

18 Une carte de ce projet est disponible sur nrlp. iwmi. org/main/maps. asp. 


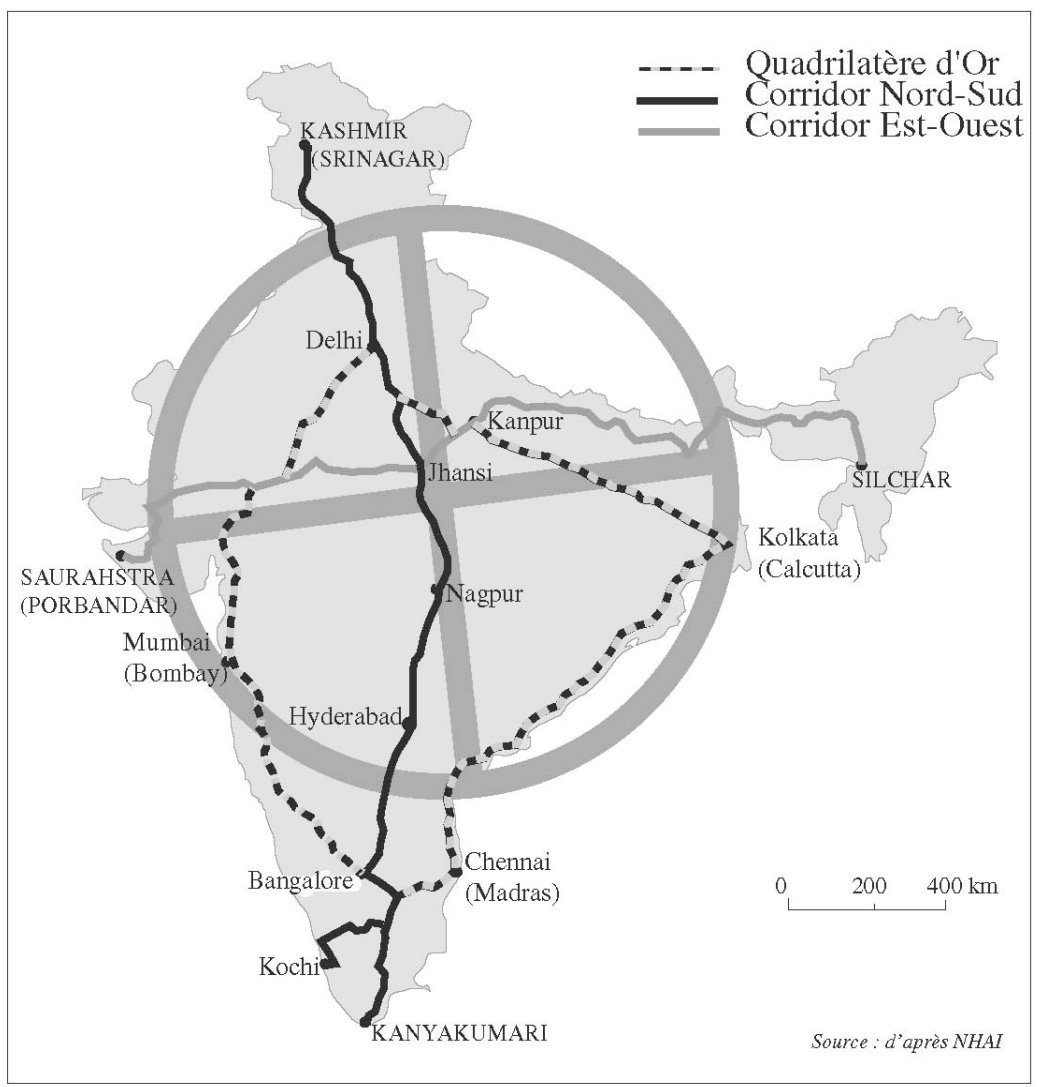

Fig. 5 Le chakra autoroutier

The chakra of expressways

Et le PDS ? On a compris que les flux de blé et de riz transportés par la Food Corporation of India dans toute l'Inde participent de la même territorialité que les flux de pèlerins ou la carte des autoroutes : il s'agit de faire circuler des grains à travers tout l'espace national pour mieux l'intégrer, dans toutes les directions possibles. Alors qu'en France le schème dominant de représentation du territoire national est fondé sur l'opposition Paris-province, qu'il s'agit de « casser » pour tenter de créer la figure idéale de l'hexagone équilibré, en Inde la représentation dominante est celle d'une roue ou d'une étoile reliant les points cardinaux.

Cette représentation est-elle si répandue cependant ? Avouons tout d'abord qu'elle est rarement explicite dans les interviews ; mais ce n'est pas pour autant qu'elle n'existe pas. Il importe de ne pas confondre la réalité géographique avec un " modèle spatial ", c'est-à-dire une représentation scientifique qui rend compte de réalités spatiales sans forcément exister explicitement dans l'esprit des acteurs (Bruneau, 2006). Plus remarquable, le PDS est d'habitude avant tout jugé par 
les personnes interrogées comme justement contraire à cette représentation. Il n'apparaît pas comme un facteur d'unité nationale, loin de là, puisqu'il permet aux forts d'opprimer les faibles, puisque les espaces déficitaires sont mal approvisionnés, puisque les États en surproduction pestent contre les autres et vice versa. Mais là encore, ce n'est pas parce que la réalité va à l'encontre de l'idéal-type que celui-ci n'existe pas. Chez les hauts fonctionnaires ou les hommes politiques, on rencontre souvent cet objectif d'un espace national à quadriller par des flux de grains, " du Kashmir à Kanya Kumari ». Chez l'agriculteur, parfois - fierté du paysan punjabi de «nourrir la nation» (Gupta, 1998); chez le consommateur illettré, rarement. Pourtant, ce jeune musulman de Hyderabad rencontré en 2005 insistait sur les deux usages de sa carte d'alimentation : pour s'approvisionner en pétrole lampant, mais aussi pour prouver son identité ( Kerosen ke lie, aur nationality ke lie »). Le PDS permet indirectement de montrer son indianité, rappelons-le, dans un milieu où celle-ci peut être contestée aux musulmans suspects d'être des immigrés. Au total, il y a fort à parier que cet attachement à un service public étatique n'est pas seulement lié au souci d'approvisionnement domestique ; il y a aussi (dans une moindre mesure) l'attachement à un service véritablement national ou du moins provincial, qui relie les individus à l'État, et par-delà à la nation. Le PDS est assurément lié à la citoyenneté. Voilà pourquoi on peut difficilement s'en passer.

\section{Conclusion}

La pesante machinerie publique qui redistribue à prix subventionnés le grain ou le sucre achetés dans les zones de surproduction connaît de nombreux dysfonctionnements. Impératifs sociaux, soucis productivistes, intérêts illicites, nombreux sont des facteurs expliquant son maintien dans une Inde qui pourtant connaît de profondes réformes libérales. Il en reste un : le PDS, dans son idéal, correspond à deux rapports au territoire national qui se superposent en grande partie. La territorialité hindoue et son interprétation par la droite nationaliste, fondée sur les points cardinaux et sur leurs liaisons, tout comme la territorialité nehruvienne, fondée sur le souci d'intégration par des flux économiques, correspondent toutes deux à une conception de l'espace national basée sur la circulation.

Aujourd'hui cependant, les représentations nehruviennes sont en perte de vitesse, c'est le moins qu'on puisse dire. Quant aux représentations hindoues, elles incluent à côté de celles évoquées plus haut une vision très décentralisée du territoire, présente dans le discours du BJP et que prônait déjà Gandhi, qui peut aller à l'encontre des pratiques territoriales jusque-là dominantes. On assiste même à une certaine revanche du localisme gandhien : la décentralisation politique, à la ville comme à la campagne, a été relancée par les amendements constitutionnels de 1992 ; la gestion participative de l'irrigation ou de la forêt se trouve au goût du jour. Tout cela correspond à un retrait de «l'intérêt national » au profit des prérogatives locales - au moins dans le discours. 
Aujourd'hui, libéralisme, victoires électorales des nationalistes hindous et morceaux choisis du vieux fond gandhien se conjuguent pour affaiblir le souci d'intégration nationale (d'intégration spatiale et même sans doute sociale). Voilà qui peut affaiblir le PDS - même si la crise alimentaire mondiale de 2006-2008 lui a récemment rendu une légitimité. C'est ainsi qu'on peut relever dans les mesures prises ces dernières années :

- l'essor des «prélèvements décentralisés » : les stocks sont alors constitués directement par les États de l'Union, ce qui décharge New Delhi d'un fardeau financier. Cette décentralisation menace l'idée même de péréquation nationale. Mais elle va dans le sens d'une autonomie des régions qui n'est pas pour déplaire au modèle de la droite hindoue des petites régions janapada, voire à celui du swaraj gandhien ;

- le développement des importations pour grossir les stocks du sud de l'Inde. Sauf années exceptionnelles, ces importations sont jugées moins coûteuses et complexes que de transporter le grain produit au nord à travers le pays : mais c'est alors la fin de flux domestiques intégrateurs. Et aucun de nos trois modèles de territorialité n'encourage une telle politique : parions donc qu'elle ne prendra guère d'ampleur ;

- depuis 2003 une grande liberté est laissée au négoce privé pour acheter les grains directement à la ferme ou dans des marchés informels : en 2005-2006, seulement $21 \%$ de la production de blé fut acheté par l'État (contre $30 \%$ en 2001-2002, année record). Cargill, Continental, mais aussi les grandes sociétés indiennes ITC ou Reliance, semblent devoir prendre une partie de la place qu'occupait la FCI pour les prélèvements sur le blé. La victime risque d'être le consommateur pauvre, si les stocks publics devenus insuffisants obligent l'État à réduire encore les distributions subventionnées. Le pari est que la croissance économique génère un développement général, et que ne se répètent point trop les flambées de prix alimentaires mondiaux. Un autre pari, plus implicite, est que le sentiment national ne pâtira pas de la disparition des flux organisés par l'État. Preuve sans doute que, désormais, la territorialité nehruvienne est moribonde : l'intégration de l'espace national, pense-t-on, se fera par les lois de l'offre et de la demande. Le danger est qu'alors demeure sans rival une territorialité nationaliste hindoue dérivant vers des processus d'exclusion religieuse et spatiale.

\author{
GECKO \\ Université de Paris-Ouest-Nanterre-La Défense \\ 200, avenue de la République \\ 92001 Nanterre Cedex \\ frederic.landy@u-paris10.fr
}

\title{
Bibliographie
}

Assayag J. (2001). L'Inde, désir de nation, Odile Jacob, 349 p.

Berti D., Tarabout G. (éd.) (2009). Territory, Soil and Society in South Asia, Delhi, Manohar-CSH. 
Brosius C. (2009). " Mapping the nation's body: Territorial processions in propaganda videos of the Hindu right ", in Berti et Tarabout (éd.), op. cit., p. 341-379.

Bruneau M. (2006). L'Asie d'entre Inde et Chine. Logiques territoriales des États, Belin, 317 p.

Bruslé T. (2006). Aller et venir pour survivre ou s'enrichir. Circulations de travail, logiques migratoires et construction du monde chez les Népalais en Inde, thèse de géographie, université de PoitiersMigrinter, 2 vol.

Chambard J.-L. (1996). " Les trois grands dieux à la porte du roi Bali. La tradition orale d'un village et notre image de l'hindouisme populaire en Inde du Nord ", in C. Champion (éd.), Traditions orales dans le monde indien, n ${ }^{\circ}$ 18, Éd. de l'EHESS, coll. "Purushartha », p. 229-272.

Chopra R.N. (1988). Food Policy in India. A Survey, New Delhi, Intellectual Publishing House.

Claveyrolas M. (2010). "Construire un espace à part. Circulation rituelle et territoire sacré ", in V. Dupont, F. Landy (éd.), Circulation et territoire dans le monde indien contemporain, Éd. de I'EHESS, coll. "Purushartha », p. 41-72.

Collignon B. (2005). "Que sait-on des savoirs géographiques vernaculaires? », Bulletin de l'Association de géographes français, 3, p. 321-331.

Deshpande S. (1993). "Imagined Economies. Styles of Nation-Building in Twentieth Century India », Journal of Arts and Ideas, 25-26, décembre, p. 4-35.

Dumont, L. (1957). Une sous-caste de l'Inde du Sud. Organisation sociale et religion des Pramalai Kallar, EPHE-Mouton, $460 \mathrm{p}$.

Feldhaus, A. (2003). Connected Places. Region, Pilgrimages and Geographical Imagination in India, Palgrave, New York, MacMillan, $322 \mathrm{p}$.

Filliozat J. (1969). « Le temps et l'espace dans les conceptions du monde indien », Revue de synthèse, 55-56, juil.-déc., p. 281-295.

Fussman G. (1980). «Le concept d'empire dans I'Inde ancienne », in M. Duverger (éd.), Le Concept d'empire, PUF, p. 379-396.

Gaborieau M. (1992). « Des dieux dans toutes les directions. Conception indienne de l'espace et classification des dieux », in V. Bouiller, G. Toffin (éd.), Classer les dieux ? Des panthéons en Asie du Sud, Éd. de l'EHESS, coll. "Purushartha » n 15, p. 23-42.

Gaenszle M., Gengnagel J. (éd.) (2006). Visualizing Space in Banaras. Images, Maps, and the Practice of Representations, Wiesbaden, Harrassowitz Verlag, 358 p.

Gandhi M. K., Collected Works of Mahatma Gandhi Online, www.gandhiserve.org

Goswami M. (2004). Producing India : From Colonial Economy to National Space, University of Chicago Press, $400 \mathrm{p}$.

Guha R. (2005). «Verdicts on Nehru. Rise and Fall of a Reputation », Economic and Political Weekly, 40, 19, p. 1958-1962.

Gupta A. (1998). Postcolonial Developments. Agriculture in the Making of Modern India, Durham, Duke University Press, $410 \mathrm{p}$.

Jackson J. B. (1994). A Sense of Place. A Sense of Time, New Haven, Yale University Press.

Jaoul N. (2007). " Dalit processions : street politics and democratization in India », in Cruise O'Brien, Donal \& Strauss, Julia (éd.), Staging Politics : Power and Performance in Asia and Africa, Londres, IB Tauris, pp.173-193.

Khilnani S. (1999). The Idea of India, New Delhi, Penguin India, 263.

Kumar G., Landy F. (2009). "Vertical governance : Brokerage, patronage and corruption in Indian metropolises », in J. Ruet, S. Tawa Lama-Rewal (éd.), Governing India's Metropolises, New Delhi, Routledge, p. 105-134.

Lachaier P. (1999). Firmes et entreprises en Inde. La firme lignagère dans ses réseaux, Karthala-IFP-EFEO, $403 \mathrm{p}$. 
Lachaier P. (à paraître). "Une vision de la bourse au curcumin (turmeric) de Sangli (Maharashtra) », in Bulletin de l'EFEO.

Landy F. (2006). Un milliard à nourrir. Grain, territoire et politiques en Inde, Belin, 270 p.

Mahendra Dev S., Kannan K.P., Ramachandran N. (éd.) (2003). Towards Food Secure India, Delhi, IHD-CESS-Manohar, $464 \mathrm{p}$.

Mooij J. (1999). Food Policy and the Indian State: The Public Distribution System in South India, Oxford University Press, Delhi, 293 p.

Naidu R. (1990). Old Cities, New Predicaments. A Study of Hyderabad, Sage, New Delhi, 177 p.

Nanda M. (2003). "Postmodernism, Hindu Nationalism and "Vedic Science" », Frontline, 20, 26, 20 déc. 2003, www.frontlineonnet.com/fl2026/stories/20040102000607800.htm

Navlakha G. (2006). «Pilgrim's Progress Causes Regression », Economic and Political Weekly, 41, 27-28, 8-15 juillet, p. 2975-2977.

Nehru J. (1946), The Discovery of India, Oxford Univ. Press, 1985, 582 p.

Ramaswamy S. (2006). " Enshrining the Map of India : Cartography, Nationalism, and the Politics of Deity in Varanasi », in M. Gaenszle et J. Gengnagel (éd.), op. cit., p. 165-190.

Savarkar V. D. (1923). Hindutva. Who is a Hindu, Veer Savarkar Prakashan, Bombay, 1969, 141 p.

Tuan Y.-F., (1976). « Geopiety : A Theme in Man's Attachment to Nature and to Place », in D. Lowenthal, M. Bowden (éd.), Geographies of the Mind, Oxford University Press, p. 11-39.

Tyagi D.S., (1990). Managing India's Food Economy. Problems and Alternatives, New Delhi, Sage, $240 \mathrm{p}$.

Van der Veer P. (1994). Religious Nationalism : Hindus and Muslims in India, Berkeley, Univ. of California Press, 247 p.

Vergati A. (2004). "Modèle hindou de ville royale. Népal, Inde du Nord (xII ${ }^{\mathrm{e}}$-xx ${ }^{\mathrm{e}}$ siècles) ", Journal des africanistes, 74 (1-2), pp.435-455.

Zins M.J. (2005). "Rites publics et deuil patriotique en Inde: les funérailles de la guerre indo-pakistanaise de 1999 ", Archives de sciences sociales des religions, 131, http://assr.revues.org/index3258.html. 